\title{
Massive haemorrhage due to ulcerative colitis presenting
} as melaena

\author{
Aristi Aristodemou, S. Ryder and M.R. Jacyna \\ Department of Gastroenterology, Northwick Park Hospital, Watford Road, Harrow, Middlesex, HAI \\ $3 U J, U K$
}

\begin{abstract}
Summary: We present a patient with known ulcerative colitis who presented with massive lower gastrointestinal bleeding in the form of melaena. Extensive investigations indicated that the source of blood loss was only from the colon, was attributable to the colitis, and settled when the colitis was treated with steroids. Melaena may be due to blood loss from the colon.
\end{abstract}

\section{Introduction}

Traditional teaching defines melaena as the passage of offensive, sticky, tarry-block stool, ${ }^{1}$ and dictates that it is usually caused by bleeding in the upper gastrointestinal tract, proximal to the colon in $95 \%$ of cases. ${ }^{2}$ Rarely however, a lesion which bleeds in the caecum may also present as melaena. ${ }^{3,4}$

Ulcerative colitis is a chronic inflammatory disorder of the colon, in which the mucosa of the large bowel, involving the rectum and extending more proximally, is inflamed. This condition usually presents with fresh, red rectal bleeding in association with diarrhoea. We report a patient with known total ulcerative colitis presenting with massive haemorrhage from the colon as melaena.

\section{Case history}

A 67 year old man presented with a 2 day history of passing liquid, black, tarry, motions, $6-8$ per day, accompanied by vague lower abdominal discomfort. The patient had been diagnosed as having ulcerative colitis 6 years previously. The diagnosis had been confirmed histologically and a barium enema at that time indicated that the entire colon was involved. A subsequent colonoscopy also confirmed a pan-colitis. The patient was initially treated with sulphasalazine, $1 \mathrm{~g}$ twice daily to which he had responded well, although more recently he had been switched to mesalazine $400 \mathrm{mg}$ thrice daily. In addition to the colitis, an abdominal aortic aneurysm had been repaired 3 years previously.

Correspondence: M. Jacyna, M.D., M.R.C.P.

Accepted: 19 February 1992
On general examination, the only abnormal clinical finding was pallor. There was no abdominal tenderness, guarding or masses. Rectal examination revealed the presence of liquid, melaena stool. No fresh red blood was seen. Haemoglobin was $6.6 \mathrm{~g} / \mathrm{dl}$. He was transfused 4 units. Rigid sigmoidoscopy was unsuccessful due to largen amounts of liquid melaena stool obscuring visiono In view of the history and clinical finding of melaena, an upper gastrointestinal endoscopy (to the distal duodenum) was performed, but no abnormality was detected. In view of his previous aortic surgery, the possibility of an aorto-enteric fistula was considered, but an aortogram showed no evidence of fistula or false aneurysm. Mesenteric angiography was also normal and did not suggest a site for his bleeding. A CT scan of the abdomen showed no abnormality, and in particular no evidence of peri-aortic inflammatory change.

Over the next 4 days, the patient continued to produce large amounts of melaena, with his haemoglobin falling repeatedly in spite of multiple (25 units) blood transfusions. A repeat upper gastrointestinal endoscopy again failed to disclose any abnormality. A chromium-51-labelled red cell scan was performed and this indicated active bleeding in the caecum extending down the entire colon. A colonoscopy was thus attempted. On entering the rectum it was immediately apparent that there was an active colitis, with black discoloration of the overlying mucosa due to altered blood. The lumen of the colon was also full of black, liquid stool, which appeared to be arising from the mucosa. No other lesion was seen and no fresh red blood was observed. Biopsies were taken and histological assessment confirmed an acutely active ulcerative colitis. A barium enema confirmed a pancolitis, but no other lesion was seen.

Oral mesalazine $400 \mathrm{mg}$ thrice daily was con- 
tinued, and he was also commenced on $40 \mathrm{mg}$ of prednisolone by mouth and made a dramatic improvement. Over a 7 day period, his melaena gradually diminished, and his bowel frequency reduced to one formed stool per day. His haemoglobin was maintained without transfusion and began to climb, and his ESR fell from 43 to 18 . He was discharged home on $7.5 \mathrm{mg}$ prednisolone and $2.4 \mathrm{~g}$ mesalazine per day. When reviewed in clinic 8 weeks after discharge he remained well with no further bleeding or diarrhoea. Repeat sigmoidoscopy revealed a quiescent, granular-looking mucosa, with no evidence of fresh or altered blood. At 6 months follow-up he remains well.

\section{Discussion}

The black colour which characterizes melaena, results from digestion of blood to produce haematin. Blood arising from the upper gastrointestinal tract will be acted upon by acid gastric juices and digestive enzymes in the duodenum and jejunum to produce this discoloration. However reports of melaena arising from proximal colonic bleeding have also been described, most owing to neoplastic lesions of the caecum or ascending colon. ${ }^{3,4}$ Other bleeding colonic lesions may also present as melaena. For example, a recent case report describes a young AIDS patient who presented with severe, uncontrolled gastrointestinal bleeding as melaena. No upper gastrointestinal cause for bleeding was identified but at operation the cause of the melaena was found to be due to severe haemorrhagic colitis, attributable to cytomegalovirus infection. ${ }^{5}$

Thus although most cases of heavy colonic bleeding present with fresh, red blood per rectum, it seems that occasionally patients may mislead clinicians and present with melaena. The reason for this probably relates to delayed transit time within the colon and bacterial degradation of blood to haematin. Experimentally, melaena from the large bowel has been produced in two studies. In the first study, melaena occurred in patients in whom more than $300 \mathrm{ml}$ blood was introduced into the caecum at the time of appendicectomy and when the transit time was greater than 24 hours. ${ }^{6}$ In the second study, it was shown that melaena occurred when transit times were greater than 14 hours. ${ }^{7}$ Both studies indicate that it is transit time and not the site of bleeding which is important in producing melaena. It is recognized that transit times are increased in ulcerative colitis ${ }^{8,9}$ thereby prolonging the fermentative action of bacteria. In the patient described in this report, there was a total colitis with evidence (from the isotope scan) of bleeding mostly from the caecum downwards. This probably explains why he presented with melaena.

Clinicians should be aware that melaena does not invariably indicate bleeding from the upper gastrointestinal tract. In patients who present with melaena, with no obvious source of blood loss identified at upper gastrointestinal endoscopy, the possibility of a colonic lesion should be considered and a colonoscopy should be strongly considered.

\section{References}

1. Isselbacher, K.J. \& Richter, J.M. Haematemesis, melaena and haematochezia. In: Petersdorf, R.G., Adams, R.D., Branwold, E., Isselbacher, K.J., Martin, J.B. \& Wilson, J.D. (eds) Harrison's Principles of Internal Medicine. McGraw-Hill, New York, 1983, pp. 199-200.

2. Shearman, D.J.C. \& Finlayson, N.D.C. Gastrointestinal bleeding. In: Diseases of the Gastrointestinal Tract and Liver. Churchill-Livingstone, Edinburgh, 1982.

3. Avery Jones, F. \& Gummer, J.W.P. Clinical Gastroenterology. Blackwell, Oxford, 1960, p. 288.

4. Vipond, M.N., Sim, A.J.W. \& Dudley, H.A.F. Colonic bleeding may cause melaena. Br J Clin Pract 1990, 44: $369-370$.

5. Chinoy, R.F. \& Pradhan, S.A. Intractable diarrhoea and melaena in an AIDS patient. Indian J Gastroenterol 1990, 9: $159-160$.

6. Luke, R.G., Lees, W. \& Rudick, J. Appearance of the stool after the introduction of blood into the caecum. Gut 1964, 5: 77-79.

7. Hilsman, J.H. The colour of blood-containing faeces following the instillation of citrated blood at various levels of the small intestine. Gastroenterology 1950, 15: 131-134.

8. Black, D.A., Ainley, C.C., Senapati, A. \& Thompson, R.P.H Transit times in ulcerative proctitis. Scand $J$ Gastroenterol 1987, 22: 872-876.

9. Chan, S.S., Reddy, S.N., Villaneuva, J. et al. Colonic motility and transit of intraluminal contents in ulcerative colitis. Gastroenterology 1989, 96: A81. 